\title{
Institutional risk factors for norovirus outbreaks in Hong Kong elderly homes: a retrospective cohort study
}

Hualiang Lin ${ }^{1 \dagger}$, Sammy Ng${ }^{2 \dagger}$, Shelley Chan ${ }^{2}$, Wai Man Chan ${ }^{2}$, Krystal CK Lee ${ }^{1}$, Suzanne C Ho ${ }^{1}$ and Linwei $\operatorname{Tian}^{1 *}$

\begin{abstract}
Background: Most of the institutional outbreaks of norovirus in Hong Kong occur in elderly homes, the proportion being $69 \%$ in 2006. Residents in elderly homes are a special population seriously affected by norovirus infections, it is necessary to investigate the risk factors of the norovirus outbreaks in Hong Kong elderly homes at the facility level.

Methods: A cohort of 748 elderly homes was followed up from January 2005 to December 2007; each elderly home was treated as one observation unit and the outcome event was the norovirus outbreak. Cox regression models were fitted to estimate the rate ratio (RR) and 95\% confidence interval (Cl) for the potential risk factors.

Results: A total of 276 norovirus outbreaks were confirmed during the study period; the outbreak rate was 12.2 (95\% Cl: 9.9-14.6) per 100 home-years; elderly homes with a larger capacity $(\mathrm{RR}=1.4,95 \% \mathrm{Cl}$ : 1.3-1.5 (per 30resident increment)), a higher staff-to-resident ratio $(R R=1.2,95 \% \mathrm{Cl}: 1.1-1.3$ (per 1/30 increment) and better wheelchair accessibility ( $\mathrm{RR}=2.0,95 \% \mathrm{Cl}$ : 1.3-3.2) were found to have an elevated norovirus outbreak rate in Hong Kong elderly homes; Elderly homes with partitions between beds had a lower rate of norovirus outbreaks (RR $=$ 0.6, 95\% Cl: 0.4-0.8).

Conclusions: Elderly home capacity, staff-to-resident ratio and wheelchair accessibility were risk factors for norovirus outbreaks in Hong Kong elderly homes. Partitions between beds were a protective factor of norovirus outbreaks. These results should be considered in the infection control in Hong Kong elderly homes.
\end{abstract}

\section{Background}

Hong Kong is facing an ageing population. In 2006, $12.4 \%$ of Hong Kong population were over 65 years old [1]. The population projection showed that the proportion of the elderly would go up to $21.9 \%$ in 2026 and $26.8 \%$ in 2033 , respectively [2]. In 2005 , there were about 60,000 elderly people residing in elderly homes, accounting for about $7 \%$ of the population aged 65 years old and above [3]. Residential care homes for the elderly are an important part of the health care system for the old age group. Evidence has shown that infection is an important cause of morbidity and mortality in the institutionalized elderly [4]. An atypically high level of

\footnotetext{
* Correspondence: linweit@cuhk.edu.hk

† Contributed equally

'School of Public Health and Primary Care, Chinese University of Hong Kong, Hong Kong SAR, China

Full list of author information is available at the end of the article
}

norovirus outbreak has been noticed in Hong Kong in early May 2006; most of the outbreaks were located in the elderly homes [5]. Healthcare-associated norovirus outbreaks are an increasingly recognized problem; better understanding of the epidemiology of these events is needed [6-8].

Norovirus is a group of closely related and highly infectious viruses which was first reported following an outbreak of gastroenteritis in Norwalk, Ohio in 1972 [9]. In recent decades, Norovirus outbreaks have presented as a growing challenge in health care facilities in many countries worldwide [10-13]. In Hong Kong, norovirus has been recognized as a major cause of both sporadic cases and outbreaks of acute gastroenteritis [14-17]. In the world, norovirus has been identified as the cause of $73 \%$ to more than $95 \%$ of nonbacterial gastroenteritis outbreaks and approximately half of all gastroenteritis outbreaks [18]. Norovirus is
C Biomed Central

C 2011 Lin et al; licensee BioMed Central Ltd. This is an Open Access article distributed under the terms of the Creative Commons Attribution License (http://creativecommons.org/licenses/by/2.0), which permits unrestricted use, distribution, and reproduction in any medium, provided the original work is properly cited. 
extremely infectious, and as few as 10 to 100 particles may be enough to initiate an infection. These viruses are also highly resistant to inactivation by freezing, heating to $60^{\circ} \mathrm{C}$, and treatment with ether, ethanol, or detergent-based cleaners. These characteristics make norovirus a major public health concern [19].

Norovirus are notoriously difficult to control in health care settings. They can affect all age groups (including both residents and staff) and an infection can be caused with a very small dose [20]. The viruses have several transmission modes, including food-borne transmission and person-to-person transmission by direct and indirect contact with vomitus or faeces from infected persons or contaminated objects. Vomitus-oral transmission may be particularly important in health care environment. There is frequently no prodrome to norovirus gastroenteritis, so an infected person may project vomit; the resulting aerosol of virus particles can be swallowed inadvertently or settle and contaminate environments $[8,21]$. Norovirus can persist in the environment for a long time, even more than 3-4 weeks, combined with its high transmissibility and high resistance to disinfectants, likely contributed to the large number of norovirus outbreaks [22]. Systematic evaluation of the institutional risk factors (staffing support, resident characteristics and environment and facilities) for norovirus outbreaks in Hong Kong elderly homes can help the design of prevention measures for norovirus outbreaks in these homes.

Most of the institutional outbreaks of norovirus in Hong Kong occurred in elderly homes, the proportion being 69\% (182 out of the total 265 episodes of outbreak) in 2006 [23,24]. Residents in elderly homes are a special population seriously affected by norovirus infections. In the nursing homes worldwide, norovirus is the most common cause of gastroenteritis and several such outbreaks have resulted in deaths due to aspiration or to exacerbations of other chronic diseases [25]. Indeed, this mild self-limited infection can lead to significant morbidity and mortality in residents of elderly homes [26].

The current study was to examine the institutional factors that may predispose to norovirus outbreaks in Hong Kong elderly homes using a retrospective cohort study, which can help the formulation of future guidelines of norovirus infection control and prevention measures at the facility level.

\section{Methods}

The cohort consisted of 748 elderly homes in Hong Kong. The baseline information on the potential predictor variables of each elderly home, in terms of resident characteristics, staffing support and environment and facility conditions, had been collected by the Territorywide Infection Control Checklist Survey in 2004, and followed up annually during 2005-2007; the survey information included home capacity (number of residents in each elderly home), percentage of residents older than 75 years old, number of nursing staff, visits by Hospital Authority Community Geriatric Assessment Team (CGAT), visits by medical officers, supply of partitions between beds, air conditioner supply, wheelchair accessibility, supply of special isolation area for infection, hygiene condition of toilets and kitchens, staff infection control training. Wheelchair accessibility was defined as whether the wheelchairs were allowed to go to other rooms within the elderly home; the hygiene condition of toilets and kitchens was assessed based on the evaluation of the survey conducted by the Elderly Health Service (EHS) of the Department of Health (DH).

In Hong Kong, the elderly homes are regulated by the Social Welfare Department (SWD) under the Residential Care Homes (Elderly Persons) Ordinance. Elderly homes in Hong Kong are run by both the private sectors and non-governmental organizations, majority of which are operating subsidized services with government subvention. According to the Codes of Practice for Residential Care Homes, all elderly homes are requested to designate an infection control officer for infectious disease prevention and control, and the outbreaks reporting in the elderly homes is mandatory, the home managers and the infection control officers are requested to report any suspected infectious disease outbreak within the elderly homes [27].

Information on the norovirus outbreaks occurring among the elderly homes during the follow-up period was extracted from the Public Health Information System (PHIS) of DH. In general, Hong Kong Depart of Health received notification of suspected outbreaks from various sources, e.g. clinicians of the hospital (if some victims were admitted to the hospital) or the staffs of the elderly homes. There is a standard protocol for outbreak management and field sampling procedures. When the notification was received, $\mathrm{DH}$ would conduct investigation by collecting epidemiological information from the victims, their relatives and staff of the elderly homes, and at the same time, stool samples were collected within 48 hours of symptom onset from all patients when initially observed and processed immediately for RNA extraction; diagnosis of norovirus infection and its quantitation were based on real-time reverse transcription-PCR assay of stool samples as described elsewhere [28]; and inspection and control measures would be implemented as well; the surveillance would only be ended if there was no more new case for 6 days from the last case. When the surveillance was over, the information would be summarized and recorded in the PHIS system, including details on first date of outbreak onset, last date of outbreak, number of persons infected, maximum, minimum and mean age of 
infected persons and the laboratory test result of each episode. The protocol of the laboratory test has been described elsewhere [5,29]. Briefly, stool specimen, food remnant, or food samples were collected during the surveillance, and tested by RT-PCR. In the current study, the "outbreak" was defined as the episode with at least two cases from a same elderly home presenting symptoms of acute onset of diarrhoea with or without vomiting in the absence of a common food source and other common symptoms include abdominal pain and fever. It is a "laboratory-confirmed norovirus outbreak" when at least one specimen was norovirus positive by laboratory testing, which was considered as an event in the current study. Homes that were closed during the follow-up were treated as censored unless there was an outbreak before the closure. Approval to conduct this study was granted by the relevant ethics committees of the Chinese University of Hong Kong (Shatin, New Territories, Hong Kong Special Administrative Region, China).

In cohort studies, subjects (elderly homes in this study) might have multiple outcome events (norovirus outbreaks in this study), which could not be assumed to be independent. The method of Wei et al. was used in this study; this method accounted for clustering in multiple failure data using robust estimates for the standard error of Cox proportional hazards [30]. However, the likelihood ratio test (LRT), used in assessing a variable's significance in multivariable models, may be invalid when standard errors are calculated by the robust method. For this reason, we examined the distribution of observed norovirus outbreaks to check clustering compared with the expected Poisson distribution, based on the observed outbreak rate (Figure 1). Outbreaks appeared to follow Poisson distribution, which lent

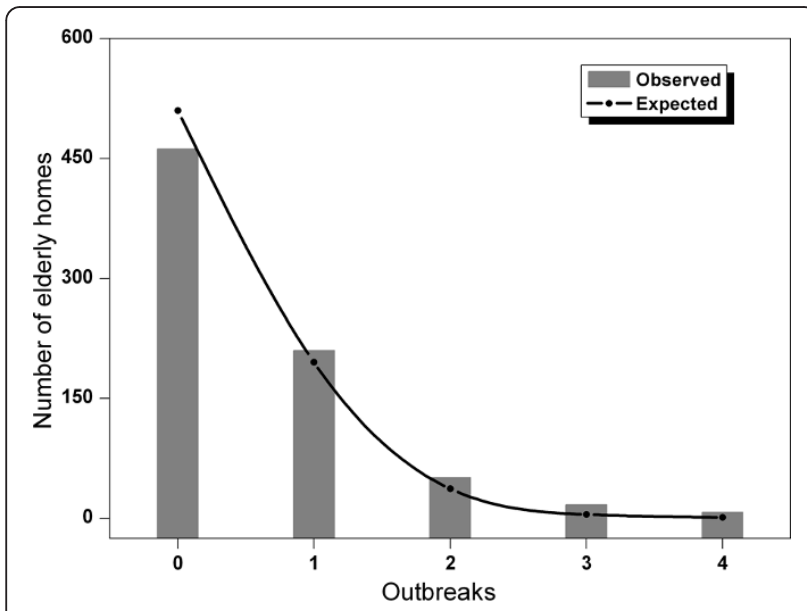

Figure 1 Distribution of observed norovirus outbreaks in Hong Kong elderly homes and expected Poisson distribution (based on rate of $\mathbf{1 2 . 2}$ outbreaks per hundred home-years). Bar: observed; line: expected. weight to the nonindependence assumption of the norovirus outbreaks among the elderly homes.

In the present study, each elderly home was treated as one observation unit and the outcome event in the Cox model was the norovirus outbreak status. The survival time was determined as the number of days from January 1, 2005 to the onset of an outbreak or the end of the study (December 31, 2007). The independent variables in the Cox regression analysis included characteristics of the elderly homes and residents, staff supporting; such as home capacity (number of residents in one home), staff-to-resident ratio, percentage of the residents older than 75 years old, percentage of bedridden residents, infection control training, visits by CGAT or medical officers, supply of isolation area and air conditioners, hygiene condition of toilets and kitchens, wheelchair accessibility, partition between beds, etc., of which the former four variables were treated as continuous variables, all others were taken as categorical variables. In the current study, some characteristics of the elderly homes might change over the follow-up period, that is, the values of some variables were not fixed over time; for example, one elder home might have 50 residents in 2005; then the number increased to 60 in 2006; and then decreased to 40 in 2007; these variables were treated as time-varying variables in the Cox regression models [31]. Univariate analysis was performed for each variable first. Variables that had a $\mathrm{p}$ value of less than 0.10 in the univariate analysis were included in the multivariate Cox regression models. If two variables were believed to be proxies for similar biologic meaning, and were highly correlated with each other, the one most significant in the univariate analysis was included in the multivariate model.

One of the main assumptions of the Cox proportional hazard model is proportionality. We checked proportionality by including time-dependent covariates in the model. Time dependent covariates are interactions of the predictors with time; in this analysis we chose to use the interactions of the finally remained variables with $\log$ (time) because this is the most common function of time used in time-dependent covariates [32]. The statistical analysis was also carried out to include the eleven "self-care hostels" in order to explore whether this exclusion has presented any potential bias. All data management and analyses were performed using SAS 9.1 (SAS Institute, Inc., Cary, North Carolina).

\section{Results}

A total of 760 elderly homes were in operation in Hong Kong in 2004, one of which was excluded because of duplicate ID number; another eleven homes, named "self-care hostels" were excluded because they were not quite comparable to other homes in terms of the 
residents' health profiles, living status, etc.; all the remaining 748 elderly homes accommodating 57,321 elderly residents were included in the final analysis.

During the study period, 276 laboratory confirmed norovirus outbreak episodes occurred in Hong Kong elderly homes, giving an overall outbreak rate of 12.2 episodes per hundred home-years (95\% CI: 9.9-14.6) or 0.004 episodes per thousand bed-days (95\% CI: 0.003 0.005). In total, 3,452 residents were infected; to ignore the outbreaks that only one resident was infected, the overall incidence was 2.0 per hundred person years $(95 \%$ CI: 1.9-2.1).

\section{Univariate analysis}

Table 1 illustrated the univariate analysis results. Norovirus outbreak rates were found to differ: homes with larger capacity, higher staff-to-resident ratio, higher fraction of older residents and better wheelchair accessibility were found to have an elevated norovirus outbreak rate; while supply of partitions between beds was found to be related to decreased rates. Percentage of bedridden residents was found to be marginally related to rates of outbreak.

No difference was found in term of staff infection control training, visits by Hospital Authority Community Geriatric Assessment Team (CGAT), visits by visiting medical officers, whether there was an isolation area for infections, whether there was air conditioner supply and the hygiene of toilets and kitchens as shown in Table 1.

\section{Multivariate model}

Significant factors identified in the univariate analysis ( $\mathrm{P}$ $<0.10$ ) were included in the multivariate Cox regression model to further explore their relationships with the norovirus outbreak rates. The proportion of bedridden residents and proportion of residents older than 75

Tables 1 Crude RRs and $95 \%$ Cls for potential risk factors related to norovirus outbreaks in Hong Kong elderly homes

\begin{tabular}{|c|c|c|c|c|c|}
\hline Factors & Home-years & Outbreaks & $\mathbf{R R}$ & $95 \% \mathrm{Cl}$ & $P$ value \\
\hline Home capacity (per 30-resident increment) & & & 1.5 & $1.4-1.6$ & $<0.0001$ \\
\hline Staff-to-resident ratio (per 1/30 increment) & & & 1.2 & $1.1-1.3$ & $<0.0001$ \\
\hline$\%$ residents older than 75 yrs (per additional 10\%) & & & 1.2 & $1.1-1.3$ & 0.0003 \\
\hline \% bedridden residents (per additional 10\%) & & & 0.9 & $0.8-1.0$ & 0.07 \\
\hline \multicolumn{6}{|l|}{ Infection control training } \\
\hline No & 1024 & 1 & 1 & & \\
\hline Yes & 8259 & 275 & 3.5 & $0.5-25.6$ & 0.2 \\
\hline \multicolumn{6}{|l|}{ Visits by CGAT } \\
\hline No & 15302 & 41 & 1 & & \\
\hline Yes & 6831 & 235 & 1.3 & $0.9-2.0$ & 0.2 \\
\hline \multicolumn{6}{|l|}{ Visits by medical officers } \\
\hline No & 267 & 14 & 1 & & \\
\hline Yes & 8094 & 262 & 0.6 & $0.3-1.1$ & 0.1 \\
\hline \multicolumn{6}{|l|}{ Isolation area } \\
\hline No & 2865 & 101 & 1 & & \\
\hline Yes & 5496 & 175 & 0.9 & $0.7-1.2$ & 0.5 \\
\hline \multicolumn{6}{|l|}{ Air conditioner supply } \\
\hline No & 157 & 12 & 1 & & \\
\hline Yes & 8204 & 264 & 0.4 & $0.2-1.1$ & 0.1 \\
\hline \multicolumn{6}{|l|}{ Hygiene condition of toilets } \\
\hline High & 113 & 2 & 1 & & \\
\hline Low & 8248 & 274 & 1.9 & $0.5-7.4$ & 0.3 \\
\hline \multicolumn{6}{|l|}{ Hygiene condition of kitchens } \\
\hline High & 438 & 13 & 1 & & \\
\hline Low & 7923 & 263 & 1.1 & $0.7-1.9$ & 0.7 \\
\hline \multicolumn{6}{|l|}{ Wheelchair accessibility } \\
\hline No & 1794 & 21 & 1 & & \\
\hline Yes & 6567 & 255 & 3.5 & $2.2-5.6$ & $<0.0001$ \\
\hline \multicolumn{6}{|l|}{ Partition between beds } \\
\hline No & 2111 & 151 & 1 & & \\
\hline Yes & 6251 & 125 & 0.25 & $0.19-0.34$ & $<0.0001$ \\
\hline
\end{tabular}


years old were believed to have similar biological meaning, in the multivariate model, proportion of residents older than 75 years old was included.

Table 2 showed the results of the multivariate model. Home capacity and staff-to-resident ratio were statistically related to elevated rates of norovirus outbreaks. In the multivariate analysis, better wheelchair accessibility was still significantly related to increased norovirus outbreak rates after adjusting for potential confounding factors $(\mathrm{RR}=2.0,95 \% \mathrm{CI}: 1.3-3.2)$; while supply of partitions between beds was still a protective factor (RR $=0.6,95 \% \mathrm{CI}: 0.4-0.8)$. And percentage of residents older than 75 years was not statistically significant after adjusting for potential confounding factors. When the eleven "self-care hostels" were included in the statistical analysis, the results remained similar.

The proportionality assumption tests for four variables were not significant either individually or collectively, so we did not have enough evidence to reject proportionality and assumed that we have satisfied the assumption of proportionality for this model.

\section{Discussion}

The current study, with an analytical epidemiology study design, has produced unique insights into the potential risk factors of norovirus outbreaks in Hong Kong elderly homes. The finding that larger elderly homes had higher risk of norovirus outbreaks was consistent with the findings of one prospective cohort study in England and one case-cohort study in New York [33,34]. The higher risk in larger elderly homes might be due to the possible difference in infection control practice, resulting in more outbreaks being discerned and reported by larger elderly homes. However, when factors indicating the infection control practices, such as isolation areas for infection, staff infection control training and hygiene of toilets and kitchens were controlled for in the multivariate Cox regression model, it was still highly associated with increased norovirus outbreaks. Thus home capacity itself

\begin{tabular}{|c|c|c|c|}
\hline Factors & RR & $95 \% \mathrm{Cl}$ & $P$ value \\
\hline Home capacity (per 30-resident increment) & 1.4 & $1.3-1.5$ & $<0.0001$ \\
\hline Staff-to-resident ratio (per 1/30 increment) & 1.2 & $1.1-1.3$ & $<0.0001$ \\
\hline$\%$ residents older than 75 (per additional 10\%) & 2.1 & $0.7-6.1$ & 0.2 \\
\hline \multicolumn{4}{|l|}{ Wheelchair accessibility } \\
\hline No & 1.0 & & \\
\hline Yes & 2.0 & $1.3-3.2$ & 0.002 \\
\hline \multicolumn{4}{|l|}{ Partition between beds } \\
\hline No & 1.0 & & \\
\hline Yes & 0.6 & $0.4-0.8$ & 0.002 \\
\hline
\end{tabular}

might lead to a true increase in the outbreaks of norovirus in Hong Kong elderly homes. Homes with more residents had a higher probability of introducing pathogens from the community as well as transmission within the homes. The relative difficulty of serving more residents within one elderly home, as well as the increased person-to-person contact among residents, their relatives, visitors and staff with different characteristics, might create higher chance of introduction and transmission of norovirus in larger homes than in smaller ones [34].

The relationship between nurse staffing and health care quality has been extensively examined in the past decades [35-43]. However, the effect of nurse staffing on the infection outbreaks in health care facilities has not been conclusive. Some previous studies [33,38,43-47] found that nurse understaffing was one potential risk factor for nosocomial infection risks; inconsistent with the previous studies, the current study found that homes with a higher staff-to-resident ratio had an elevated risk of norovirus outbreaks. A similar relationship was also observed in a study of respiratory infection in Hong Kong elderly homes by a previous analysis, which found that homes with nurse support had higher risk of respiratory infections [48]. These findings might have also suggested another possible norovirus transmission mode, "attendant-borne transmission" [49]. While tending to patients, the health care staff might inadvertently carry pathogens from one resident to another; they may do this by direct contact through their hands or indirectly through their use of health care facilities. Such attendant-borne transmission has been reported as the major route for some hospital-acquired pathogens [50], and might be the case in the current study, as in the 276 outbreaks, health care staff were infected in about half $(132 / 276)$ and low hand washing compliance has been reported in Hong Kong health care workers [51].

The higher risk among homes with higher staff-toresident ratio might be explained in part by the fact that pathogens could be introduced by the nursing staff from outside community. The nursing staff usually has lower probability to be infected, partly because they are less vulnerable than the older population [5], and they may have generated some immunity to the organisms. The wide activity range of the nursing staff in the elderly homes enabled them to serve as a transmission vector among the residents, and elderly homes and outside community, as reported in one hospital outbreak, norvirus was detected in the asymptomatic staff [52].

Elderly homes with better wheelchair accessibility had elevated risk of norovirus outbreaks. The underlying mechanism remains unknown. Increased mobility of the residents may result in higher chance of norovirus transmission within the elderly home. Residents themselves 
and the wheelchair surfaces might play an important role in facilitating the transmission, either by direct or indirect contacts, as contaminated surfaces have been reported to be an important transmission vector in some norovirus outbreaks $[53,54]$. It was also possible that this variable has served as an indicator for other risk factors, such as different characteristics of the residents; the health status or age structure, etc. might be different among homes with or without better wheelchair accessibility, which might contributed to different risk of norovirus transmission.

Partition between beds was found to be linked to reduced risk of norovirus outbreaks in Hong Kong elderly homes. Previous studies have showed that roommate exposure and neighborhood exposure are important risk factors for the transmission of diarrhea infection in health care facilities [36,37]. It was believed that the mechanisms of protective effects of partitions between beds might be related to that of these two exposures; to some extent, supply of partitions between beds could yield an immediate benefit, providing a physical barrier that limited the transfer of infection between the two areas [55]; and thus reduced the risks caused by roommate and neighborhood transmission.

There are a number of limitations to be considered when interpreting the results of the current study. The accuracy of the data obtained is of critical importance. Our outcome information was collected by an outbreak surveillance system; it was largely affected by the completeness of the reporting; under-reporting was a limitation of the current study. It was usually the larger outbreaks that received attention and were reported, and thus included in the study. The present study excluded eleven "self-care hostels", which might be one potential source of selection bias; however, when we included those homes in our analysis, which gave us a very similar results; so if the exclusion presented bias, it should not have been very serious. One should also be noted that it was an institutional-level study; some individual-level characteristics that might play an important role in the norovirus outbreaks were not taken into consideration [56], further investigations were needed to confirm the findings from the current study.

\section{Conclusions}

In summary, this study evaluated the impact of institutional risk factors on norovirus outbreaks in Hong Kong elderly homes. Based on the present results, it is recommended that increased emphasis be placed on proper infection control measures, especially in homes with a larger capacity, a higher number of nursing staff, a higher staff-to-resident ratio, better wheelchair accessibility; partitions between beds should be considered as an effective infection control measure in elderly homes.

\section{Acknowledgements}

This study was supported by the Hong Kong Research Fund for the Control of Infectious Diseases, Health and Health Services Research Fund, Health Services Research Fund, Health Care \& Promotion Fund (RFCID/HHSRF/HSRF/ HCPF, Grant No. 08070452). We thank Dr. SS Lee from Chinese University of Hong Kong for helpful discussions and comments.

\section{Author details}

${ }^{1}$ School of Public Health and Primary Care, Chinese University of Hong Kong, Hong Kong SAR, China. ${ }^{2}$ Department of Health, Hong Kong SAR, China.

\section{Authors' contributions}

LWT, SN, SCH and KL conceived and designed the study; HLL, SC, WMC and LWT performed the data management and data analysis; $\mathrm{HLL}, \mathrm{SCH}, \mathrm{KL}$ and LWT drafted the manuscript. All authors contributed to the revision of the manuscript and approved the submitted version of the manuscript.

\section{Competing interests}

The authors declare that they have no competing interests.

Received: 11 August 2010 Accepted: 11 May 2011

Published: 11 May 2011

\section{References}

1. Tse W, Leung E: Vital Statistics in 2006. Public Health and Epidemiology Bulletin 2007, 16.

2. Census 2001 Report. Census and Statistics Department, HKSAR.

3. Social Welfare Department: Residential care services for the elderly.[http:// www.swd.gov.hk/tc/index/site_pubsvc/page_elderly/sub_residentia/], accessed on April 30, 2007.

4. Jiang X, Turf E, Hu J, Barrett E, Dai XM, Monroe S, Humphrey C, Pickering LK, Matson DO: Outbreaks of gastroenteritis in elderly nursing homes and retirement facilities associated with human caliciviruses. J Med Virol 1996, 50(4):335-41.

5. Ho ECM, Cheng PKC, Lau AWL, Wong AH, Lim WWL: Atypical Norovirus Epidemic in Hong Kong during Summer of 2006 Caused by a New Genogroup II/4 Variant. J Clin Microbiol 2007, 45(7):2205-11.

6. Lopman BA, Reacher MH, Vipond IB, Hill D, Perry C, Halladay T, Brown DW, Edmunds WJ, Sarangi J: Epidemiology and cost of nosocomial gastroenteritis, Avon, England, 2002-2003. Emerg Infect Dis 2004, 10(10):1827-34.

7. Meakins SM, Adak GK, Lopman BA, O'Brien SJ: General outbreaks of infectious intestinal disease (IID) in hospitals, England and Wales, 19922000. J Hosp Infect 2003, 53(1):1-5.

8. Lopman BA, Adak GK, Reacher MH, Brown DW: Two epidemiologic patterns of norovirus outbreaks: surveillance in England and wales, 1992-2000. Emerg Infect Dis 2003, 9(1):71-7.

9. Kapikian AZ, Wyatt RG, Dolin R, Thornhill TS, Kalica AR, Chanock RM: Visualization by immune electron microscopy of a 27-nm particle associated with acute infectious nonbacterial gastroenteritis. J Virol 1972, 10(5):1075-81.

10. Richards CL: Infection control in long-term care facilities. J Am Med Dir Assoc 2007, 8(3):S18-S25.

11. Kirk MD, Veitch MG, Hall GV: Gastroenteritis and food-borne disease in elderly people living in long-term care. Clin Infect Dis 50(3):397-404.

12. Dai YC, Xia M, Zhan HC, Liu Y, Li JD, Chen Q, Yu SY, Nie J, Farkas T, Jiang X: Surveillance and risk factors of norovirus gastroenteritis among children in a southern city of China in the fall-winter seasons of 2003-2006. J Paediatr Child Health 46(1-2):45-50.

13. Ryan MJ, Wall PG, Adak GK, Evans HS, Cowden JM: Outbreaks of infectious intestinal disease in residential institutions in England and Wales 19921994. J Infect 1997, 34(1):49-54

14. Lau CS, Wong DA, Tong LKL, Lo JYC, Ma AMC, Cheng PKC, Lim WWL: High rate and changing molecular epidemiology pattern of norovirus 
infections in sporadic cases and outbreaks of gastroenteritis in Hong Kong. Journal of Medical Virology 2004, 73(1):113-17.

15. Cheng PKC, Wong DKK, Chung TWH, Lim WWL: Norovirus contamination found in oysters worldwide. Journal of Medical Virology 2005, 76(4):593-97.

16. Chan MCW, Sung JJY, Lam RKY, Chan PKS, Lee NLS, Lai RWM, Leung WK: Fecal viral load and norovirus-associated gastroenteritis. Emerging Infectious Diseases 2006, 12(8):1278-80.

17. Ho ECM, Cheng PKC, Wong DA, Lau AWL, Lim WWL: Correlation of norovirus variants with epidemics of acute viral gastroenteritis in Hong Kong. Journal of Medical Virology 2006, 78(11):1473-79.

18. Atmar RL, Estes MK: The epidemiologic and clinical importance of norovirus infection. Gastroenterology Clinics of North America 2006, 35(2):275-90.

19. Dolin R: Noroviruses - challenges to control. New England Journal of Medicine 2007, 357(11):1072-73.

20. Goodgame R: Norovirus gastroenteritis. Curr Gastroenterol Rep 2006, 8(5):401-08

21. Marks PJ, Vipond IB, Carlisle D, Deakin D, Fey RE, Caul EO: Evidence for airborne transmission of Norwalk-like virus (NLV) in a hotel restaurant. Epidemiol Infect 2000, 124(3):481-7.

22. US Center for Disease Control and Prevention: Outbreaks of gastroenteritis associated with noroviruses on cruise ships-United States, 2002. MMWR 2002, 51(49):1112-5.

23. Kung K, Law Y, Lee CC, Lau A, Low K, Kong W: 2006 year in review. Communicable Diseases Watch 2007, 4(2):5-7.

24. SCEIFD: Update on Noroviral Gastroenteritis Situation in Hong Kong Scientific Committee on Enteric Infections and Foodborne Diseases, Centre for Health Protection; 2007, 1-5.

25. Estes MK, Prasad BW, Atmar RL: Noroviruses everywhere: has something changed? Current Opinion in Infectious Diseases 2006, 19(5):467-74

26. Bradley SF: Double, double toil and trouble: Infections still spreading in long-term-care facilities. Infection Control and Hospital Epidemiology 2005, 26(3):227-30.

27. Hong Kong SAR: Residential Care Homes (Elderly Persons) Regulation, Chapter. 459A, Section. 181997.

28. Chan MC, Sung JJ, Lam RK, Chan PK, Lee NL, Lai RW, Leung WK: Fecal viral load and norovirus-associated gastroenteritis. Emerg Infect Dis 2006, 12(8):1278-80

29. Tsang OTY, Wong ATY, Chow CB, Yung RWH, Lim WWL, Liu SH: Clinical characteristics of nosocomial norovirus outbreaks in Hong Kong. Journal of Hospital Infection 2008, 69(2):135-40.

30. Wei $L$, Lin $D Y$, Wiessfeld $L$ : Regression analysis of multivariate incomplete failure time data by modelling marginal distributions. J Am Stat Assoc 1989, 84:1065-73.

31. Boeck S, Haas M, Laubender RP, Kullmann F, Klose C, Bruns CJ, Wilkowski R, Stieber P, Holdenrieder S, Buchner H, Mansmann U, Heinemann V: Application of a time-varying covariate model to the analysis of CA 19-9 as serum biomarker in patients with advanced pancreatic cancer. Clin Cancer Res 2010, 16(3):986-94.

32. Introduction to SAS: UCLA: Academic Technology Services, Statistical Consulting Group.[http://www.ats.ucla.edu/stat/sas/faq/test_proportionality. $\mathrm{htm}$ ], [cited November 24, 2007].

33. Lopman BA, Andrews N, Sarangi J, Vipond IB, Brown DWG, Reacher MH: Institutional risk factors for outbreaks of nosocomial gastroenteritis: survival analysis of a cohort of hospital units in South-west England, 2002-2003. J Hosp Infect 2005, 60(2):135-43.

34. Li J, Birkhead GS, Strogatz DS, Coles FB: Impact of institution size, staffing patterns, and infection control practices on communicable disease outbreaks in New York State nursing homes. Am J Epidemiol 1996 143(10):1042-9.

35. Mark BA: Methodological issues in nurse staffing research. West I Nurs Res 2006, 28(6):694-709.

36. Aiken LH, Smith HL, Lake ET: Lower Medicare mortality among a set of hospitals known for good nursing care. Med Care 1994, 32(8):771-87.

37. Cho SH, Ketefian S, Barkauskas VH, Smith DG: The effects of nurse staffing on adverse events, morbidity, mortality, and medical costs. Nurs Res 2003, 52(2):71-9.

38. Kovner C, Gergen PJ: Nurse staffing levels and adverse events following surgery in U.S. hospitals. Image J Nurs Sch 1998, 30(4):315-21.
39. Kovner C, Jones C, Zhan C, Gergen PJ, Basu J: Nurse staffing and postsurgical adverse events: an analysis of administrative data from a sample of U.S. hospitals, 1990-1996. Health Serv Res 2002, 37(3):611-29.

40. Lichtig LK, Knauf RA, Milholland DK: Some impacts of nursing on acute care hospital outcomes. J Nurs Adm 1999, 29(2):25-33.

41. Mark BA, Harless DW, McCue M, Xu Y: A longitudinal examination of hospital registered nurse staffing and quality of care. Health Serv Res 2004, 39(2):279-300.

42. Needleman J, Buerhaus P, Mattke S, Stewart M, Zelevinsky K: Nurse-staffing levels and the quality of care in hospitals. N Engl J Med 2002, 346(22):1715-22.

43. Unruh L: Licensed nurse staffing and adverse events in hospitals. Med Care 2003, 41(1):142-52.

44. Haley RW, Bregman DA: The role of understaffing and overcrowding in recurrent outbreaks of staphylococcal infection in a neonatal specialcare unit. J Infect Dis 1982, 145(6):875-85.

45. Fridkin SK, Pear SM, Williamson TH, Galgiani JN, Jarvis WR: The role of understaffing in central venous catheter-associated bloodstream infections. Infect Control Hosp Epidemiol 1996, 17(3):150-8.

46. Archibald LK, Manning ML, Bell LM, Banerjee S, Jarvis WR: Patient density, nurse-to-patient ratio and nosocomial infection risk in a pediatric cardiac intensive care unit. Pediatr Infect Dis J 1997, 16(11):1045-8.

47. Stegenga J, Bell E, Matlow A: The role of nurse understaffing in nosocomial viral gastrointestinal infections on a general pediatrics ward. Infect Control Hosp Epidemiol 2002, 23(3):133-6.

48. Wong WY: Epidemiology of the infection outbreaks among the residential care homes for the elderly (RCHEs) in Hong Kong and the associated risk factors of the respiratory infection outbreaks in 2005 (MPH thesis). School of Public Health Chinese University of Hong Kong; 2007, 1-34.

49. Ewald PW: Evolution of virulence. Infect Dis Clin North Am 2004, 18(1):1-15.

50. Ewald PW: Evolution of infectious disease. New York: Oxford University Press; 1994.

51. Lam BC, Lee J, Lau YL: Hand hygiene practices in a neonatal intensive care unit: a multimodal intervention and impact on nosocomial infection. Pediatrics 2004, 114(5):e565-71.

52. Gallimore $\mathrm{Cl}$, Cubitt D, du Plessis N, Gray JJ: Asymptomatic and symptomatic excretion of noroviruses during a hospital outbreak of gastroenteritis. J Clin Microbiol 2004, 42(5):2271-4.

53. Cheesbrough JS, Green J, Gallimore Cl, Wright PA, Brown DWG: Widespread environmental contamination with Norwalk-like viruses (NLV) detected in a prolonged hotel outbreak of gastroenteritis. Epidemiol Infect 2000, 125(1):93-98.

54. Barker J, Vipond IB, Bloomfield SF: Effects of cleaning and disinfection in reducing the spread of Norovirus contamination via environmental surfaces. Journal of Hospital Infection 2004, 58(1):42-49.

55. Cath N, Andrew S: Engineering Solutions for Infection Control. ANSYS Advantage 2007, 1(2):S8-9.

56. Fretz R, Svoboda P, Schorr D, Tanner M, Baumgartner A: Risk factors for infections with Norovirus gastrointestinal illness in Switzerland. Eur J Clin Microbiol Infect Dis 2005, 24(4):256-61.

\section{Pre-publication history}

The pre-publication history for this paper can be accessed here: http://www.biomedcentral.com/1471-2458/11/297/prepub

doi:10.1186/1471-2458-11-297

Cite this article as: Lin et al: Institutional risk factors for norovirus outbreaks in Hong Kong elderly homes: a retrospective cohort study. BMC Public Health 2011 11:297. 